\section{Use of Floor Polish in Mounting Sections for Light Microscopy}

\author{
Kevin M. Imel
}

Washington State University and University of Idaho
The latter problem can be compensated for, if necessary, by mounting sections individually under small cover slips.

Floor polish has been shown to be suitable for use as a mounting media with the following properties:

1. Tissue integrity (at least for those tissues examined) is considerably better

Over the years a number of methods have been described to mount tis- maintained than with either sucrose or Permount $\mathrm{t}^{\circ}$.

sue sections on glass slides (for example see Humason $1979^{1}$ ). This paper 2. Background florescence is lower than either sucrose or Permount $t^{\circ}$.

presents a novel mounting medium that is inexpensive and readily available at 3 . Since floor polish is a aqueous based medium it is suitable for use when a the nearest grocery store - floor polish. This floor polish is available under a medium containing organic solvents is not desirable.

variety of trade names (e.g. Brite and Future ${ }^{\circ}$, both from S.C. Johnson and 4 . Floor polish cures rapidly. If warmed at $70^{\circ} \mathrm{C}$, the mounting is ready to be Son, Racine, WI) labeled for use with "no-wax" floors. It has been found that viewed within 20 to 30 minutes.

this product is well suited to use as a mounting medium for both 5 . Mountings are stable over time.

gelatin/albumin embedded sections as well as epoxy sections. The floor pol- 6 . Floor polish is inexpensive and readily available.

ish has demonstrated a much lesser degree of tissue distortion in some tis-

sues as compared to the mounting media previously used in our laboratory, including Permount ${ }^{\circ}$ (Fisher Chemical, Fair Lawn, NJ) and $30 \%$ sucrose in distilled $\mathrm{H}_{2} \mathrm{O}$.

While only Brite floor polish was thoroughly examined in this study, preliminary results indicate that Future is equally suited. It is likely that many of the other brands of floor polish suitable for no-wax floors would also be suitable for use as a mounting medium.

It should be noted that neither the author nor family have any financial inter-

The sciatic nerve of an adult rat was fixed in $4 \%$ paraformaldehyde and embedded in a mixture of gelatin and albumin. The tissue block was then hardened in the same fixative, rinsed in $0.1 \mathrm{M} \mathrm{PO}_{4}$ buffer and cryoprotected with $30 \%$ sucrose solution in $0.1 \mathrm{M} \mathrm{PO}_{4}$ buffer. Sections were cut at $40 \mathrm{~mm}$ with a cryostat. These sections were then stained with a monoclonal antibody series and reacted with horseradish peroxidase (these sections were part of a separate study and have incidentally been used as demonstrations for this paper). No other histological stains were used on these sections.

Cover slips were then attached to each of the dried slides using one of three mounting media: 1) Permount ${ }^{\circ}$, 2) $30 \%$ sucrose in deionized $\mathrm{H}_{2} \mathrm{O}$, and 3) Brite ${ }^{\circ}$ floor polish. The sections to be used with Permount were air dried and then dehydrated through an ethanol ( $40 \%$ through $95 \%)$ and butyl alcohol $(100 \%)$ series followed by a rinse in xylene. These sections were then immediately mounted with Permount ${ }^{\circ}$. The sections to be mounted with sucrose were air dried and then layered on $30 \%$ sucrose for 5 to 10 minutes and then mounted in $30 \%$ sucrose; The coverslips were sealed with dental wax. The sections to be mounted in Brite ${ }^{\circ}$ were mounted by partially air drying and then mounting with the floor polish. Air drying the sections completely causes the sections to become temporarily opaque but has little effect on the overall tissue integrity in the final preparation. Partial air drying was used simply to avoid this opaque phase.

The mounting media were allowed to cure and the slides were then observed under standard light microscopy as well as with appropriate optics for both fluorescein and rhodamine fluorescent dyes.

\section{Results \& Discussion}

As can be seen in figures $1 \mathrm{a}$ (Permount) and $1 \mathrm{~b}$ (sucrose), the tissue has undergone a dramatic distortion with large fractures forming within the nerve fiber bundle and the epineurium (connective tissue sheath) has pulled away form the outside of the bundle (arrows mark the boundaries of the displaced capsule). The section in figure $1 \mathrm{c}$ (floor polish) shows some fracturing of the bundle, but it is distorted to a far lesser degree than either the Permount ${ }^{\circ}$ or sucrose and the epineurium has not been displaced.

Permount ${ }^{2}$, sucrose, and floor polish were also compared for autofluorescence when viewed under appropriate optics for fluorescein and rhodamine dyes. The Permount ${ }^{2}$ medium yielded a high background autofluorescence, the sucrose a noticeable autofluorescence, and the floor polish negligible autofluorescence.

The floor polish mounting would appear to be quite stable over time. Slides containing gelatin/albumin sections have been stored for approximately two years and epoxy sections for approximately 30 months with no visible degradation of the floor polish medium. It should be noted that these times are in no way an end point; but simply reflect the results to date. In any case, floor polish is considerably more stable than sucrose which tends to begin drying out almost immediately.

Floor polish as a mounting media does suffer from at least two weakness: 1) since it is an aqueous solution, sections stained with water soluble dyes quickly become de-stained, and 2) the sections tend to migrate during the curing process, even if mounted on subbed slides, if warmed to speed curing. ests in S.C. Johnson and Son, Inc. Products used in this study was by chance.

1. Humason, G.L. 1979. Animal Tissue Techniques. Fourth ed. W.H. Freeman \& Co. San Francisco, CA. 98-110.

The author wishes to thank Dr. Mark DeSantis and Mr. J. Franklin Bailey for their helpful comments on this manuscript.
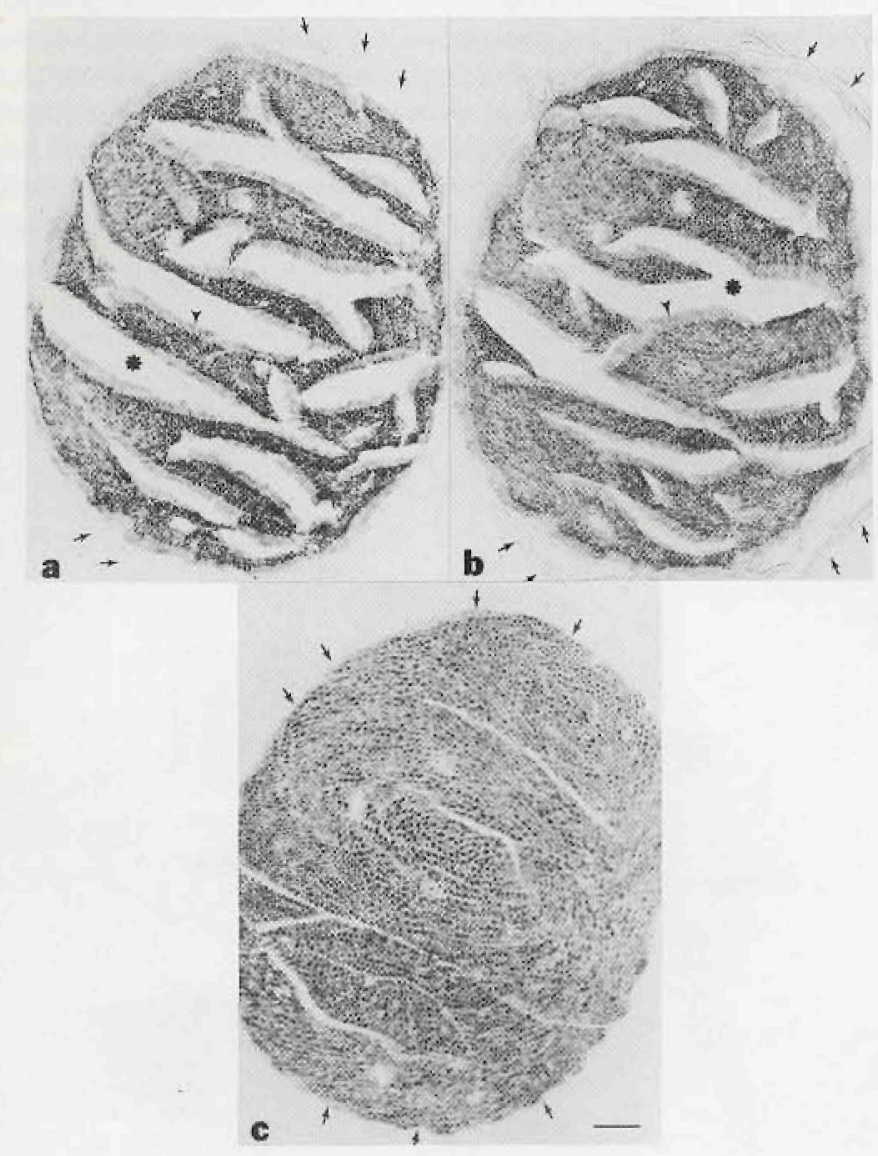

Firgure 1. Adult rat sciatic nerve, embedded in gelatinalbumin and reacted with substrate for horseradish peroxidase (HRP) after incubation with an antibody series the second of which was conjugated to HRP Sections were mounted in Permount (a) $30 \%$ sucrose (b) and Brite fioor polish (c). Large fractures seen in (a) and (bb) are absent in (c). Arrows mark the boundary of the epineurium that has been displaced from the nerve bundle during the mounting process. Representative fractures in (a) and (b) are marked with an asterisk. The columar structures lining the fractures (arrow heads) are sections of axons that have fallen and are now laying on their sides. The dark circular profiles seen in the sections are axons to which the monoclonal antibody has bound. No ther histological stain was uned on these sections. Scale bar $=100 \mu m$ 


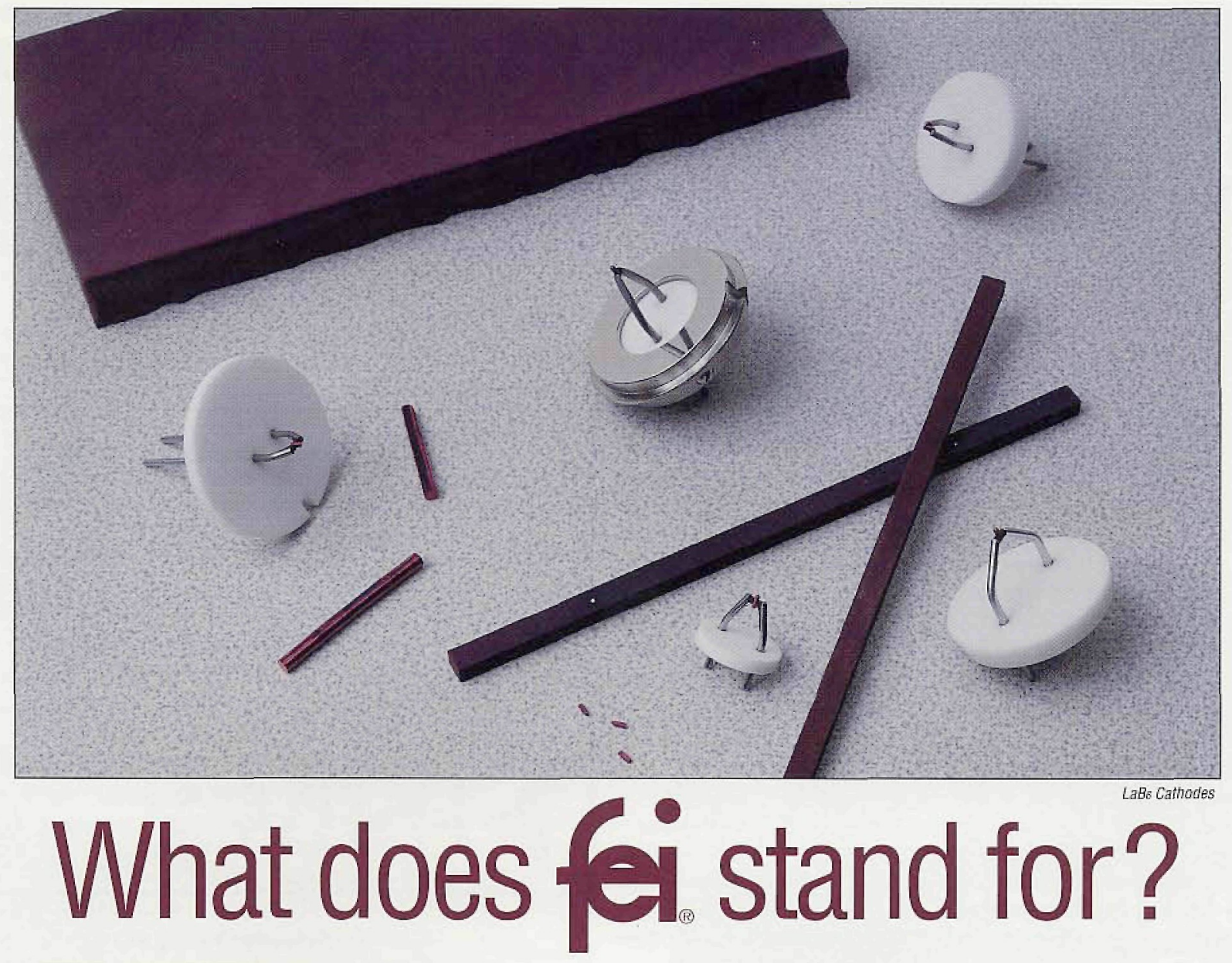

FEl's Mini Vogel Mount was the first true long-life, high stability LaB6 cathode for all electron beam instruments. The MVM is the economical emitter choice, providing consistent high quality performance and the best cost-per-use value. FEI is the innovator in the application of field emission technology...

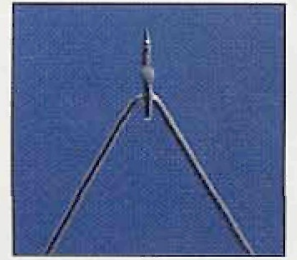

Field Emission Cathodes

FEI supplies Schottky field emitters to EM manufacturers worldwide. Schottky emission's high current intensity has established it as the preferred electron source for high resolution SEM, TEM, Auger, ESCA, EDX, and lithography.

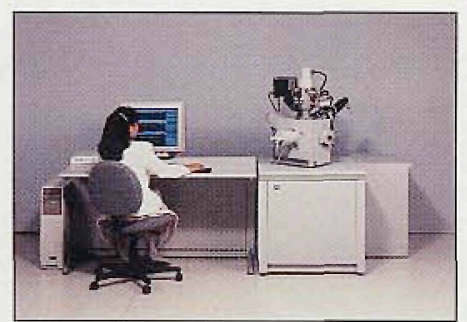

\section{FIB Workstations}

Focused ion beam micromilling workstations range from the 8"-wafer compatible model to

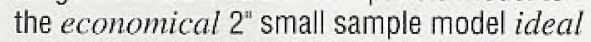
for semiconductor, biological, TEM, and MEMS specimens.

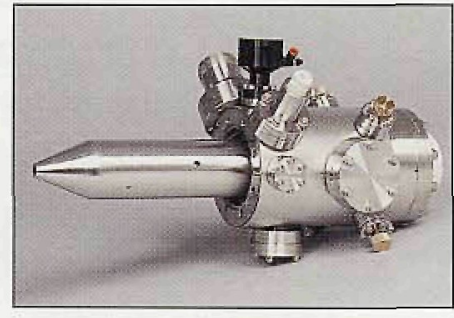

UHV Electron and lon Columns FEI's high current density, UHV field emission focusing columns easily install on existing SIMS, Auger, and SEM instruments.

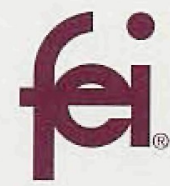

FEI Company

7451 NE Evergreen Parkway

Hillsboro, OR $97124-5830$

(503) 640-7500 Fax (503) 640-7509

email:dlh@feico.com

Now, when you think of FEI, you'll know we are the Specialists in Field Electron and lon Technology. 